\title{
Optimization of positrons generation based on laser wakefield electron acceleration
}

\author{
Yuchi Wu, ${ }^{1,2}$ Dan Han, ${ }^{1}$ Tiankui Zhang, ${ }^{1}$ Kegong Dong, ${ }^{1}$ Bin Zhu, ${ }^{1}$ \\ Yonghong Yan, ${ }^{1}$ and Yuqiu $\mathrm{Gu}^{1,2}$ \\ ${ }^{1}$ Science and Technology on Plasma Physics Laboratory, Research Center of Laser Fusion, CAEP, \\ Mianyang, Sichuan 621900, China \\ ${ }^{2}$ IFSA Collaborative Innovation Center, Shanghai Jiao Tong University, Shanghai 200240, China \\ (Received 23 April 2015; revised manuscript received 11 June 2016; published 23 August 2016)
}

\begin{abstract}
Laser based positron represents a new particle source with short pulse duration and high charge density. Positron production based on laser wakefield electron acceleration (LWFA) has been investigated theoretically in this paper. Analytical expressions for positron spectra and yield have been obtained through a combination of LWFA and cascade shower theories. The maximum positron yield and corresponding converter thickness have been optimized as a function of driven laser power. Under the optimal condition, high energy $\left(>100 \mathrm{MeV}\right.$ ) positron yield up to $5 \times 10^{11}$ can be produced by high power femtosecond lasers at ELI-NP. The percentage of positrons shows that a quasineutral electron-positron jet can be generated by setting the converter thickness greater than 5 radiation lengths.
\end{abstract}

DOI: 10.1103/PhysRevAccelBeams.19.081303

\section{INTRODUCTION}

Since the chirped pulse amplification technique was proposed [1], there has been a large increase in the number of short-pulse, high-intensity laser facilities in the world. With these high intensity lasers, high-charge-density relativistic positron beams can be obtained from laser-plasma interactions [2-12]. These beams provide a new pathway to produce antiparticles, have potential as injectors in electron-positron colliders for high energy particle physics study, or generate relativistic pair-plasmas in the laboratory for astrophysics research $[13,14]$.

In recent years, there have been many theoretical and experimental studies on laser positron generation [2-12, 15-20]. Experimentally, positron beams can be generated from two different configurations. Irradiating high $\mathrm{Z}$ solid targets with high-peak-power, high-energy lasers is a direct way to generate positrons. Using this method, experiments were carried out by Chen's group on petawatt, picosecond laser facilities at Lawrence Livermore National Laboratory, University of Rochester and Osaka University [4-8]. Owing to the target rear surface sheath field, the positrons behave as a quasi-monoenergetic beam with peak energy several $\mathrm{MeV}$ to tens of $\mathrm{MeV}$ and $10^{11}-10^{12}$ yield per shot. Higher energy positrons can be obtained based on laser wakefield electron acceleration (LWFA). Sarri et al. [9-11] adopted low energy ( 1-10 J) femtosecond lasers interacting with underdense plasma

Published by the American Physical Society under the terms of the Creative Commons Attribution 3.0 License. Further distribution of this work must maintain attribution to the author(s) and the published article's title, journal citation, and DOI. to accelerate electrons to several hundreds of $\mathrm{MeV}$. The positrons are produced when the accelerated electrons pass through a secondary high $\mathrm{Z}$ converter. The positron spectrum presents a continuous distribution, and the highest energy can also reach several hundreds of $\mathrm{MeV}$. The positron yield is about $10^{8}$ per shot.

Essentially, positrons were generated from high energy particles propagating in matter. Although there are two main processes to create positrons: the "trident process" and the "Bethe-Heitler $(\mathrm{BH})$ process." The trident process is normally ignored when relativistic particles pass a thick target, because the cross section of the $\mathrm{BH}$ process is much larger than that of the trident process [21]. Laser-induced, high-energy electrons propagation in high $\mathrm{Z}$ targets to generate positrons with the $\mathrm{BH}$ process is similar to cascade showers in cosmography. Combing laser plasma interaction with shower theory can help us to understand laser positron production and design optimal experimental conditions. Within this framework, Myatt et al. studied positron generation from kilojoule-class, high-intensity lasers irradiating solid targets [16]. According to their research, a positron (mean energy about $1 \mathrm{MeV}$ ) yield of $5 \times 10^{11}$ can be reached with a $2.5 \mathrm{~kJ}$ laser energy on OMEGA EP, and energetic conversion of laser to positrons is about $10^{-5}$. Under this condition, the electrons from laser solid target interaction obey a Maxwellian distribution with an effective temperature of several $\mathrm{MeV}$ to several tens of $\mathrm{MeV}$. But in LWFA, the electron beams show different properties: quasimonoenergetic structure with peak energies of several hundreds of $\mathrm{MeV}$ to several $\mathrm{GeV}$, low emittance, and low energy spread [22-26]. Due to those large differences, the optimal condition cannot be extended to LWFA-based positron production directly. 
In this study, we have performed a theoretical optimization for LWFA-based positron generation. We discuss some characteristics of the positron beams generated in LWFA configuration, and include spectrum, total yield under different laser power, optimal conditions for maximum yield, and concentration in positron-electron mixed beams. Analytic approximate expressions of positron spectrum and yield were deduced form cascade shower frame, can be used to predict characteristics of positron and avoid inconvenient Monte Carlo simulations or numerical calculations.

The paper is organized as follows: In Sec. II, we present the beam property of high energy electron emission from LWFA and the basic theory of pair production with cascade shower. In Sec. III, the results are presented and discussed, and the optimal positron yield under different laser conditions is deduced. In Sec. IV, the conclusions are given.

\section{ELECTRONS EMITTED FORM LWFA AND POSITRONS PRODUCTION IN CONVERTER}

When an ultraintense and ultrashort laser pulse interacts with an underdense plasma, the ponderomotive force of the focused laser pulse will create a longitudinal plasma wave that propagates in the wake of the laser pulse with a phase velocity close to the speed of light in vacuum [27,28]. This plasma wave can provide a large accelerating electric field of several hundred gigavolts per meter [29-31]. Electrons trapped in the wakefield should reside within the focusing and accelerating phase of the wave and can obtain energy from the plasma wave field. Laser-driven plasma accelerators operate in several regimes depending on the intensity of the driving laser pulse, the density of the plasma, and the duration of the laser pulse relative to the plasma period. Nowadays, the "Bubble" regime is predominant in the LWFA research area because it can lead to a quality electron beam with high peak energy, low energy spread, low divergence, and short duration. In most LWFA experiments, laser intensity was usually chosen to $a_{0} \approx 1-3$ to make accelerating process continuously and stably. According to the 3D nonlinear theory proposed by W. Lu [32-34], a matched condition of laser plasma interaction can be designed beginning from $k_{p} w_{0}=2 \sqrt{a_{0}}$, where $k_{p}$ is wave number of plasma and $w_{0}$ is laser focal spot size. Under this optimum condition, the energy gain $\Delta E$ and total electron number $N$ can be predicted by a scaling law:

$$
\begin{gathered}
\Delta E[\mathrm{GeV}] \approx 3.8\left(\frac{P}{P_{c}}\right)^{-2 / 3} \frac{P[T W]}{100}, \\
N \approx 2.5 \times 10^{9} \frac{\lambda_{0}[\mu \mathrm{m}]}{0.8} \sqrt{\frac{P[\mathrm{TW}]}{100}},
\end{gathered}
$$

where $P$ is the laser power, $P_{c}=17 \omega_{0}^{2} / \omega_{p}^{2}[\mathrm{GW}]$ is the critical power for relativistic self-focusing [35], $\lambda_{0}$ is the laser wavelength. High quality, high energy electron beams predicted in theory were already represented in many experiments. In typical LWFA experiments, femtosecond laser pulses with an energy of several joules and peak intensity of $10^{18}-10^{19} \mathrm{~W} / \mathrm{cm}^{2}$ interact with a large-scale plasma provided by a few-mm-long gas jet or a few-cm-long capillary and gas cell with the plasma density in the range of $10^{18}-10^{19} \mathrm{~cm}^{-3}$. High quality electron beams can be reproduced steadily with peak energies from several hundreds of $\mathrm{MeV}$ to several $\mathrm{GeV}, 1 \%$ level energy spread, and $1 \pi \mathrm{mm}$ mrad transverse emittance [22-26]. High energy positrons can be generated through the propagation of these electron beams in a solid converter. For high-energy electrons, there is only a little difference in the electromagnetic process cross sections within a small energy spread range. We can treat a high quality electron beam accelerated by laser wakefield within the "Bubble" regime as a highly collimated monoenergetic beam.

The production of positrons by high energy electrons passing through a high- $Z$ converter can be regarded as a cascade shower problem, which has been researched for many years [36-38] in cosmic ray studies. When high energy electrons pass through matter, large numbers of secondary electrons and photons are generated by collision and radiation. The secondary photons further materialize into pairs by the $\mathrm{BH}$ process, which increases the number of secondary electrons and positrons. As the process goes on, more and more electrons lose their energy and give rise to pairs. In practice, electrons are incident almost normal to the target surface and their energies are much higher than the electron rest energy. According to the cross section of electrons and photons [39-41], the angles of emission or scattering of secondary electrons and photons are very small for high energy particles. We can therefore treat the shower as a longitudinal development along the direction of the incident particles and neglect the path increase due to angular spread. Then, the influence of shower development on the target thickness and material was determined. In order to remove the material dependence from the shower problem, the radiation length, $R L$, was used as the length unit in general, which corresponds to the average transition length needed for a high energy electron to decrease its energy to $1 / e$ by radiation. In our calculations, a modified radiation length expression was used to correct the effect of atomic electrons and Born's approximation [42].

$$
\frac{1}{R L}=4 a \frac{N_{A}}{A} Z(Z+1) r_{e}^{2} \ln \left(183 Z^{-\frac{1}{3}}\right) /\left[1+0.12\left(\frac{Z}{82}\right)^{2}\right]
$$

Here, $a$ is the electron fine structure constant, $r_{e}$ is the classical radius of the electron, $N_{A}$ is Avogadro's number, and $Z$ and $A$ are, respectively, the charge and mass number of the material. For lead which were used in several 
experiments, the radiation length $R L=6.52 \mathrm{~g} / \mathrm{cm}^{2}$, and corresponding thickness is $5.75 \mathrm{~mm}$.

Considering the cross sections of each processes in high energy conditions [39-41], we can ignore the Compton scattering of photons and accede collision by the continuous slowing-down approximation (CSDA). The distribution functions of electrons $f_{e_{-}}(E, t)$, positrons $f_{e+}(E, t)$, and photons $f_{p}(E, t)$ are continually changing with increasing transmission length $t$ in the target. In each step, the change of particle number can be expressed as follows (one can find more detail in Refs. [11,12,36-38,42]). Here, we distinguish the real particles as electrons and positrons, and give their own distribution.

$$
\begin{gathered}
\frac{\partial f_{e-}(E, t)}{\partial t}=-f_{e-}(E, t) \mu_{\mathrm{rad}}(E)+\int_{E}^{\infty} f_{p}\left(E^{\prime}, t\right) \phi_{\mathrm{pair}}\left(E^{\prime}, E\right) d E^{\prime}+\int_{E}^{\infty} f_{e-}\left(E^{\prime}, t\right) \phi_{\mathrm{rad}}\left(E^{\prime}, E\right) d E^{\prime}+\frac{\partial\left[f_{e-}(E, t) \varepsilon(E)\right]}{\partial E} \\
\frac{\partial f_{e+}(E, t)}{\partial t}=-f_{e+}(E, t) \mu_{\mathrm{rad}}(E)+\int_{E}^{\infty} f_{p}\left(E^{\prime}, t\right) \phi_{\mathrm{pair}}\left(E^{\prime}, E\right) d E^{\prime}+\int_{E}^{\infty} f_{e+}\left(E^{\prime}, t\right) \phi_{\mathrm{rad}}\left(E^{\prime}, E\right) d E^{\prime}+\frac{\partial\left[f_{e+}(E, t) \varepsilon(E)\right]}{\partial E} \\
\frac{\partial f_{p}(E, t)}{\partial t}=-f_{p}(E, t) \mu_{\mathrm{pair}}(E)+\int_{E}^{\infty}\left[f_{e-}\left(E^{\prime}, t\right)+f_{e+}\left(E^{\prime}, t\right)\right] \phi_{\mathrm{rad}}\left(E^{\prime}, E\right) d E^{\prime}
\end{gathered}
$$

In each formula, the first term describes the decrease of particle number and the other term accounts for the increase. $\mu_{\mathrm{rad}}(E)$ is the total probability per radiation length that a particle with energy of $E$ will radiate a photon. $\mu_{\text {pair }}(E)$ is the total pair production probability per radiation length by a photon with energy $E$. $\phi_{\text {rad }}\left(E^{\prime}, E\right)$ is the probability per radiation length that a particle with energy $E^{\prime}$ generates a new photon with energy $E$. $\phi_{\text {pair }}\left(E^{\prime}, E\right)$ is the probability per radiation length that a photon with energy $E^{\prime}$ product a new particle with energy of $E$. The expressions of these cross sections can be found in many publications [43-45].

For particle energies higher than the critical energy $\varepsilon$ [42] (an electron with energy $\varepsilon$ that loses all its energy by collision in one radiation length. As reference, the critical energy $\varepsilon \approx 7 \mathrm{MeV}$ for lead), we can treat the CSDA energy loss $\varepsilon(E)$ as a constant equal to the critical energy $\varepsilon$.

We can use asymptotic formulas for complete screening to describe the probabilities of these effects in the high energy situation. A set of equations can be obtained as follows:

$$
\begin{aligned}
\frac{\partial f_{e-}(E, t)}{\partial t}=-\int_{0}^{1}\left[f_{e-}(E, t)-\frac{1}{1-v} f_{e-}\left(\frac{E}{1-v}, t\right)\right] \varphi_{\mathrm{rad}}(v) d v+\int_{0}^{1} f_{p}\left(\frac{E}{v}, t\right) \varphi_{\mathrm{pair}}(v) d v+\varepsilon_{0} \frac{\partial f_{e-}(E, t)}{\partial E}, \\
\frac{\partial f_{e+}(E, t)}{\partial t}=-\int_{0}^{1}\left[f_{e+}(E, t)-\frac{1}{1-v} f_{e+}\left(\frac{E}{1-v}, t\right)\right] \varphi_{\mathrm{rad}}(v) d v+\int_{0}^{1} f_{p}\left(\frac{E}{v}, t\right) \varphi_{\mathrm{pair}}(v) d v+\varepsilon_{0} \frac{\partial f_{e+}(E, t)}{\partial E} \\
\frac{\partial f_{p}(E, t)}{\partial t}=-\mu_{0} f_{p}(E, t)+\int_{0}^{1}\left[f_{e-}\left(\frac{E}{v}, t\right)+f_{e+}\left(\frac{E}{v}, t\right)\right] \varphi_{\mathrm{rad}}(v) \frac{d v}{v} \\
\varphi_{\mathrm{rad}}(v)=\frac{1}{v}\left[1+(1-v)^{2}+\left(\frac{2}{3}-2 b\right)(1-v)\right] \\
\varphi_{\mathrm{pair}}(v)=v^{2}+(1-v)^{2}+\left(\frac{2}{3}-2 b\right) v(1-v), \\
\mu_{0}=\frac{7}{9}-\frac{b}{3} .
\end{aligned}
$$

$v$ is the energy fraction of the new particles, $\varphi_{\text {rad }}(v)$ and $\varphi_{\text {pair }}(v)$ are approximate expressions of the electron radiation and pair production, respectively. Adopting some mathematical techniques $[36,38,43]$, we can find analytic approximate solutions of the distribution functions. Here, we only concern electron incidence as initial condition. An expression of positrons distribution function $f_{e+}\left(E_{0}, E, t\right)$ can be written as 


$$
\begin{aligned}
f_{e+}\left(E_{0}, E, t\right) d E & =\frac{1}{(8 \pi)^{1 / 2}} \frac{J_{1}(s)}{\left[\lambda_{1}^{\prime \prime}(s) t\right]^{1 / 2}} \frac{\left(E_{0}\right)^{s}}{\left[u_{1}(s, \varepsilon / E) E\right]^{s+1}} \exp \left[\lambda_{1}(s) t\right] d E+k \frac{1}{(8 \pi)^{1 / 2}} \frac{J_{3}(s)}{\left[-A^{\prime \prime}(s) t\right]^{1 / 2}} \frac{\left(E_{0}\right)^{s}}{\left[u_{1}(s, \varepsilon / E) E\right]^{s+1}} \exp [-A(s) t] d E \\
J_{1}(s) & =\frac{B(s) C(s)}{\left[\lambda_{1}(s)-\lambda_{2}(s)\right]\left[\lambda_{1}(s)+A(s)\right]}, \quad J_{3}(s)=\frac{B(s) C(s)}{\left[\lambda_{1}(s)-\lambda_{2}(s)\right]\left[\lambda_{2}(s)+A(s)\right]} \\
t & =-\frac{1}{\lambda_{1}^{\prime}(s)} \ln \left(\frac{E_{0}}{E}\right)
\end{aligned}
$$

The expression for total number of electrons and positrons $N_{e+}\left(E_{0}, E, t\right)$ is

$$
\begin{aligned}
N_{e+}\left(E_{0}, E, t\right)= & \frac{1}{(8 \pi)^{1 / 2}} \frac{1}{s} \frac{J_{1}(s)}{\left[\lambda_{1}^{\prime \prime}(s) t+\left(1 / s^{2}\right)\right]^{1 / 2}} \frac{\left(E_{0}\right)^{s}}{\left[\rho_{1}(s, \varepsilon / E) E\right]^{s}} \exp \left[\lambda_{1}(s) t\right] \\
& +k \frac{1}{(8 \pi)^{1 / 2}} \frac{1}{s\left[-A^{\prime \prime}(s) t+\left(1 / s^{2}\right)\right]^{1 / 2}} \frac{\left(E_{0}\right)^{s}}{\left[\rho_{1}(s, \varepsilon / E) E\right]^{s}} \exp [-A(s) t] \\
t= & -\frac{1}{\lambda_{1}^{\prime}(s)} \ln \left(\frac{E_{0}}{E}-\frac{1}{s}\right)
\end{aligned}
$$

$E_{0}$ is the energy of the incident electron. Explanation of the terms not listed here, such as $\lambda_{1}, \lambda_{2}, A(s), B(s)$, $C(s), C(s), u_{1}(s, \varepsilon / E)$, and $\rho_{1}(s, \varepsilon / E)$ can be found in Ref. [38] and [42]. $k$ is a correction constant that performs well by setting $k=1 / 2$. One should notice that the approximate solutions are limited by the complete screening and CSDA approximations, which are valid for $2 \varepsilon<E \ll E_{0}$ [38].

\section{ANALYSIS OF THE POSITRON GENERATION CALCULATIONS}

\section{A. Characteristics of positron differential spectrum}

From Eq. (10), we can calculate an approximate solution of the positron differential spectrum for the incident electron energy of $E_{0}$ at a certain transmission length $t$. The spectrum can be also obtained by numerical evaluation of Eqs. (7)-(9). To access the reliability of the two methods, we also examine the result by the Monte Carlo simulations of $500 \mathrm{MeV}$ electrons propagated in lead by Geant 4 . As shown in Fig. 1, the three spectra are consistent in the low energy range (the spectra were normalized to the number of incident electrons). As the energy increasing, the approximate solution gives higher positron numbers. The percentage of the high-energy positrons is very small, which is less than 5\%, and does not obstruct us researching the problem using the approximate solutions. As indicated in Ref. [43], if the transmission length is not too small, the structure of the differential spectra is approximately satisfied by power functions, and almost does not change as the transmission length.

\section{B. Characteristics of positron yield}

The number of positrons with energy between $E_{0}$ to $E$, can be obtained both by Eq. (11) and numerical method.
Both the methods give similar results for positron yield when a high-energy electron propagates in solid matter. We find that the variation of positron number is mainly related to $E_{0} / E$. Although the incident electron energy $E_{0}$ is different, the same $E_{0} / E$ delivers a similar variation of the positron yield. From Eq. (11), we can also see the righthand side of the equation mainly concerned with $E_{0} / E$. As shown in Fig. 2, if the transmission length is not too small, the approximate solution can provide a practicable result. The maximum positron yield in energy range $\left[E_{0}, E\right]$ and its corresponding converter thickness can be also obtained from the curve. Compared with the Geant 4 simulations, both methods give higher results. In our opinion, these deviations between methods can be attributed to two

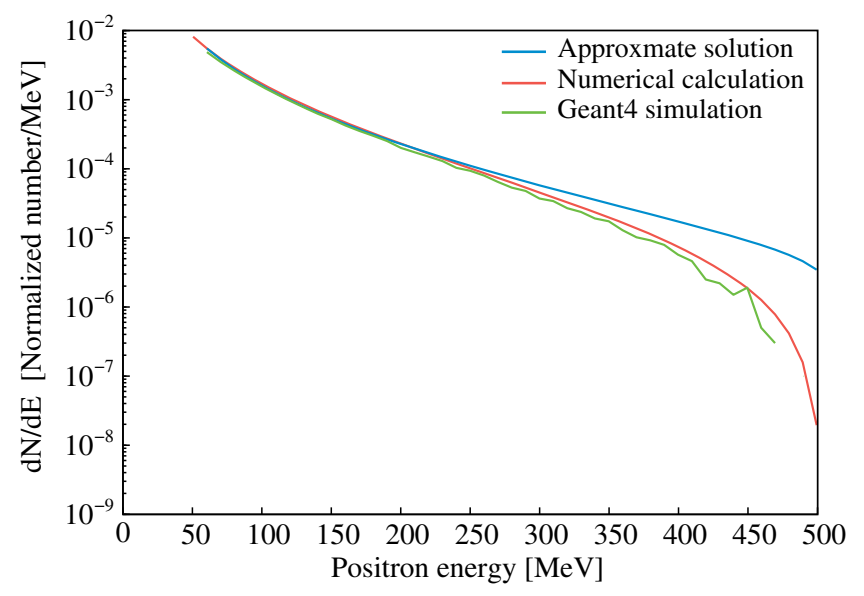

FIG. 1. Comparison of differential spectra obtained by different methods at three radiation lengths. The red curve was calculated by Eq. (8), the blue curve was obtained by a numerical method, and the green curve was simulated by Geant 4 . The energy of the incident electron is $500 \mathrm{MeV}$ and the transmission length is $3 R L$. 


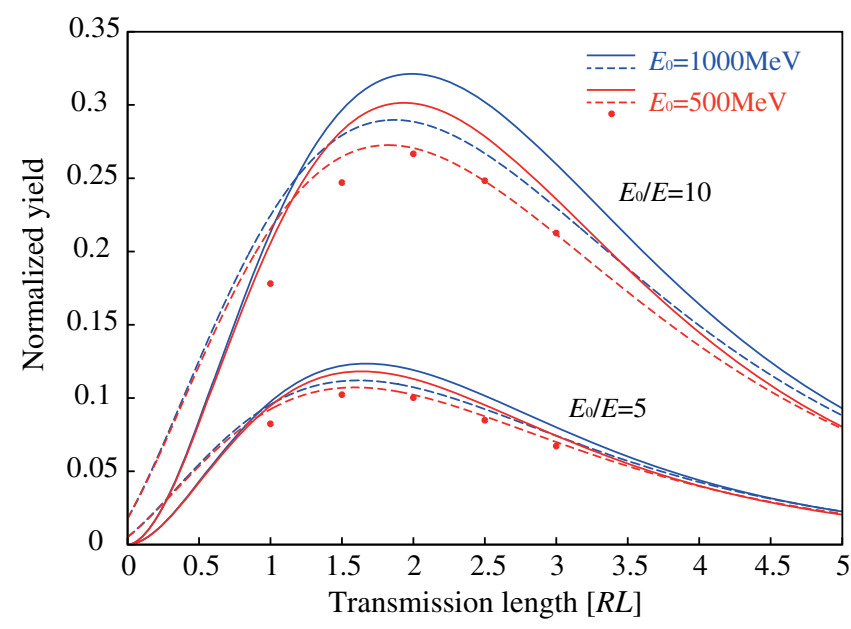

FIG. 2. Comparison of positron yields obtained by different methods with incident energies of $500 \mathrm{MeV}$ and $1000 \mathrm{MeV}$. The dash lines were calculated by approximate solution of Eq. (9), the continuous curves were obtained by a numerical method, and the dots were simulated by Geant 4 .

reasons. First, we use the completed screen approximation for cross section calculation; second, the angular dispersion was ignored in the particle propagation.

\section{Dependence of positron yields and optimal thickness on LWFA conditions}

Figure 3(a) shows the electron energy and yield obtained from scaling Eq. (1) and Eq. (2) as functions of laser power. In the calculation, we choose the laser intensity $a_{0}=3$, and the optimal condition of LWFA can be obtained from W. Lu's theory. Then, we can predict the maximum positron yield which can be obtained for a given laser power and corresponding optimal converter thickness, as shown in Fig. 3(b). In practice, people always attend to the positron yield beyond a certain energy $E$, here we consider positron energy $E \geq 100 \mathrm{MeV}$. High energy positron yield is almost linearly increased with the driven laser power. Nowadays, PW ultrashort lasers have been demonstrated in several laboratories, such as PULSER at APRI [46] or Texas Petawatt Laser [47]. Using these powerful lasers, the electron beam from LFWA can be accelerated to $10 \mathrm{GeV}$, and electron number will be beyond $10^{10}$. Positron yield will also be beyond $10^{10}$ for an optimal converter thickness near $4 R L$. With the practical possibility of high-intensity short-pulse lasers with laser power reach to $10 \mathrm{PW}$ in the near future (e.g., the "ELI-NP" facility which is under construction [48]), high energy positron yield can extend to $5 \times 10^{11}$, about 500 times higher than present results. Under this condition, high density electron-positron pair plasma with particle density reach to $10^{18} \mathrm{~cm}^{-3}$ maybe created in laboratory [12].

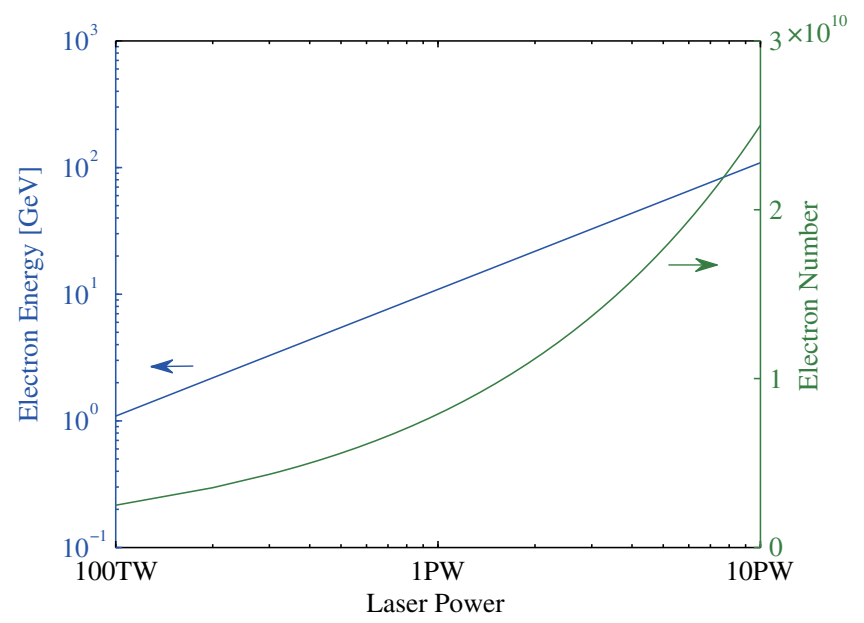

(a)

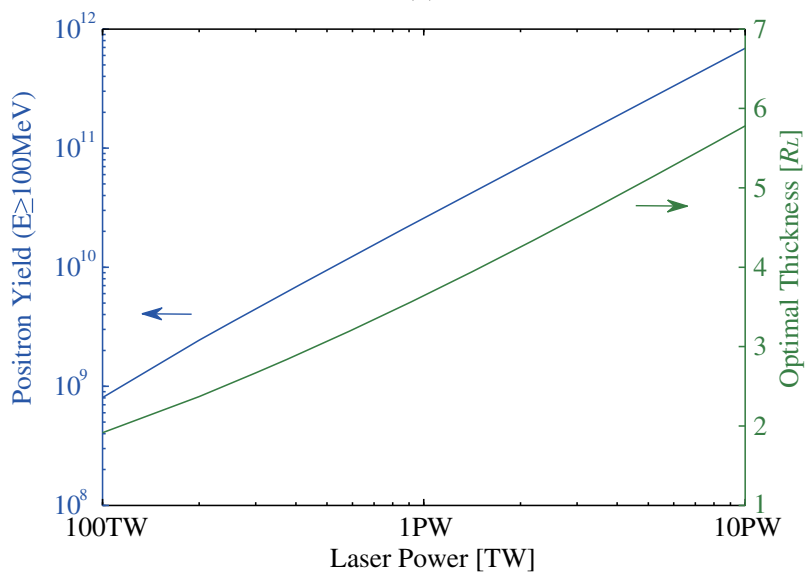

(b)

FIG. 3. (a) Electron energy (blue line) and total electron yield (green line) with different laser power under optimal LWFA conditions based on W. Lu's theory. (b) Maximum positron yield with energy beyond $100 \mathrm{MeV}$ (blue line) and corresponding optimal converter thickness (green line) as functions of driven laser power.

Experimentally, LWFA results maybe deviate from the theoretical optimal conditions. The high energy electron beams present wide range continuous spectra, which cannot be treated as monoenergetic beams in positrons production. However, we can divide an electron beam with continuous spectrum into several segments and predict characteristics of positrons by our theoretical approximate solution. As shown in Fig. 4(a), we divide the electron beam in Sarri's experiment [12] into 20 segments. Each segment is regarded as a monoenergetic electron sub-beam whose energy adopts the average energy in same energy range and same total electron number. Positron spectra and total yield can be both obtained by summing the results of monoenergetic segments, calculated by Eq. (10) and Eq. (11). Figures 4(b) and 4(c) show the good agreements of positron spectra in experiment and theory for different converter thickness. Total positron yield predicted by 
(a)

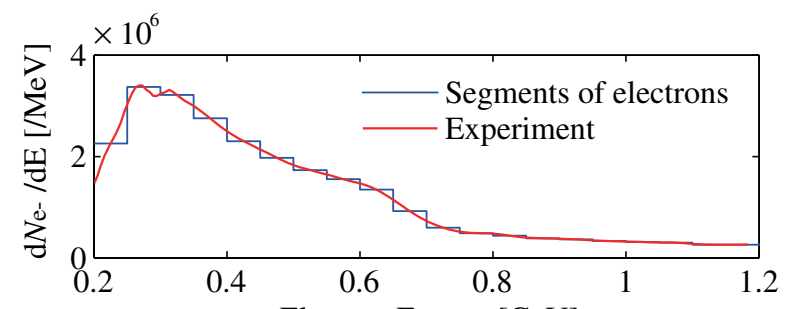

(b)

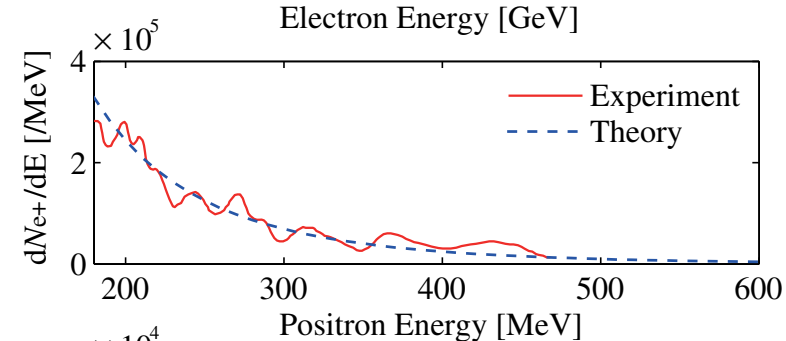

(c)

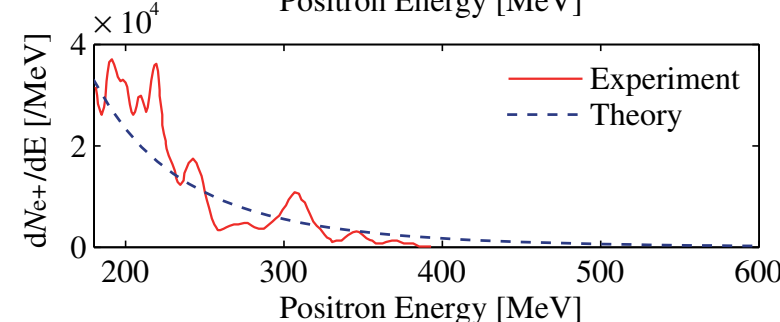

(d)

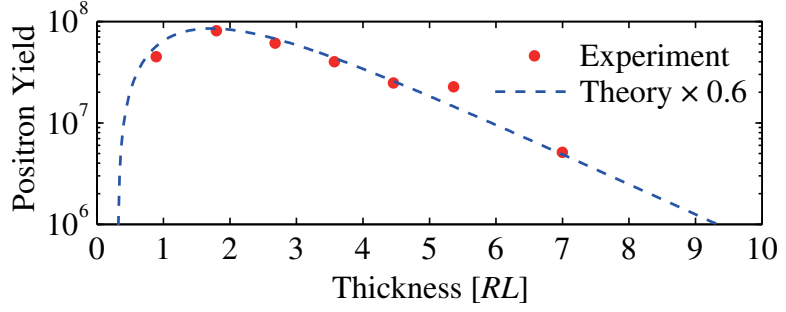

FIG. 4. Comparison of positron spectrum and total yield obtained in experiment and calculated by our theoretical expressions. Experimental results come from Ref. [12]. In the calculation, the experimental electron spectrum [red curve in (a)] was divided into 20 segments [blue curve in (a)]. (b) and (c) show the positron spectra (energy $>120 \mathrm{MeV}$ ) from experiment (red lines) and theory (blue dash lines) for different converter thickness $2 \mathrm{~cm}(3.48 R L)$ and $4 \mathrm{~cm}(6.96 R L)$. (d) shows the measured (red dots) and calculated (blue dash line) total yields as a function of converter thickness.

theory [blue dash line in Fig. 4(d)] shows the same trend of experimental result but much higher in quantity. If we add a correctional factor of about 0.6 which adopted 0.75 in Sarri's numerical calculation [12], the theory and experiment are matched well.

\section{Quasineutral electron-positron jets}

The generation of high density and high energy electronpositron beams is a basic problem in astrophysics, which is similar to jets of long gamma-ray bursts. In our study, a quasineutral electron-positron beam can be reached by an ultrashort laser. In cosmic ray theory, the total number of electrons and positrons $N_{\mathrm{e} \pm}\left(E_{0}, E, t\right)$ can be obtained by Eq. (2.100) in Ref. [37]. Comparing the expression with the

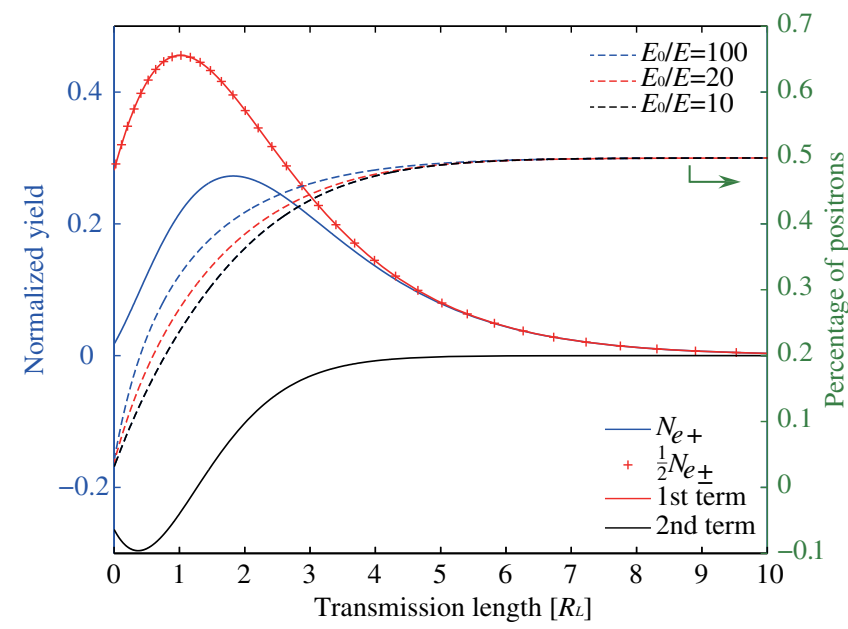

FIG. 5. Comparison of the total electric particle number $N_{\mathrm{e} \pm}\left(E_{0}, E, t\right)$ and positron number $N_{e+}\left(E_{0}, E, t\right)$ obtained by approximate solutions. In the calculation, the incident energy $E_{0}=500 \mathrm{MeV}$ and the lower bound energy $E=50 \mathrm{MeV}$. The blue curve shows the total number of positrons. The red symbol "+" shows the half number of total particles and the red curve shows the 1st term of Eq. (11) which consist with each other. The black curve shows the 2nd term of Eq. (11) and tends to zero. The dash lines show the numerical percentage of positrons with different $E_{0} / E$.

positron yield Eq. (11), we find the first term of Eq. (11) equals to $N_{\mathrm{e} \pm}\left(E_{0}, E, t\right) / 2$, as shown in Fig. 5. The second term is a correction to the positron yield, which goes to zero as the transmission length increasing. This means that the electrons and positrons will always tend to be equal as the beams propagate through the target material. We write $\eta$ as the ratio of the second term and the first term of Eq. (11).

$\eta=k \frac{J_{3}(s)}{J_{1}(s)}\left[\frac{\lambda_{1}^{\prime \prime}(s) t+\left(1 / s^{2}\right)}{-A^{\prime \prime}(s) t+\left(1 / s^{2}\right)}\right]^{1 / 2} \exp \left\{\left[-A(s)-\lambda_{1}(s)\right] t\right\}$

The percentage of positron in mixed beams can be written as $\frac{1+\eta}{2}$. Figure 5 shows that the fractions are always close to $50 \%$ if the transmission length is beyond $5 R L$, even though the $E_{0} / E$ are different. Therefore, if the transmission length is large enough, a quasineutral electron-positron beam can be obtained in a LWFA-based scheme. This condition of the neutral jet generation was consistent with previous experiments by Sarri et al. [9-12].

\section{CONCLUSION}

Positron generation based on laser wakefield electron acceleration was investigated. The energy and number of electrons produced by LWFA was determined by the scaling laws of W. Lu's theory. The positron production considered as monoenergetic electron beams propagating in a high $Z$ converter. According to the cascade shower 
theories, the analytic expressions of positron spectrum and yield were obtained, which were checked by Monte Carlo simulations and numerical calculations.

If the transmission length is large enough, the positrons always present a continuous spectrum and can be approximated satisfactorily by power functions. For a given electron beam with energy $E_{0}$, the positron yield beyond a certain energy $E$ is dependent mainly on $E_{0} / E$. The maximum positron yield and corresponding converter thickness have been optimized as a function of driven laser power in LWFA. Under our optimal condition, a high energy positron yield up to $5 \times 10^{11}$ can be produced on ELI-NP. Even if the electron beam represents continuous spectra in LWFA experiments, we can treat it as a series of monoenergetic sub-beams, and also predicted the positron generation exactly.

The percentages of positrons were calculated from approximate solutions. The percentage displays always tend to $50 \%$ as the transmission length increases. A quasineutral electron-positron jet can be easily obtained when the transmission length is beyond $5 R L$.

To estimate positron spectra and yield, we limit all the particles in the shower to linear propagation and use the full screen approximation for cross section calculations. Mount Carlo simulations show that those assumptions make the results slightly higher but still reliable.

\section{ACKNOWLEDGMENTS}

This work was supported by the Sciences and Technology on Plasma Physics Laboratory at CAEP (Grant No. 9140C680302130C68242) and the National Natural Science Foundation of China (Grants No. 11174259, No. $11375161 \&$ No. 11405159).

[1] D. Strickland and G. Mourou, Compression of amplified chirped optical pulses, Opt. Commun. 56, 219 (1985).

[2] C. Gahn, G. D. Tsakiris, G. Pretzler, K. J. Witte, C. Delfin, C. G. Wahlstrom, and D. Habs, Generating positrons with femtosecond-laser pulses, Appl. Phys. Lett. 77, 2662 (2000).

[3] C. Gahn, G. D. Tsakiris, G. Pretzler, K. J. Witte, P. Thirolf, D. Habs, C. Delfin, and C. G. Wahlstrom, Generation of $\mathrm{MeV}$ electrons and positrons with femtosecond pulses from a table-top laser system, Phys. Plasmas 9, 987 (2002).

[4] T. E. Cowan et al., High energy electrons, nuclear phenomena and heating in petawatt laser-solid experiments, Laser Part. Beams 17, 773 (1999).

[5] H. Chen, M. Nakai, Y. Sentoku, Y. Arikawa, H. Azechi, S. Fujioka, C. Keane, S. Kojima, W. Goldstein, B. R. Maddox, N. Miyanaga, T. Morita, T. Nagai, H. Nishimura, T. Ozaki, J. Park, Y. Sakawa, H. Takabe, G. Williams, and Z. Zhang, New insights into the laser produced electronpositron pairs, New J. Phys. 15, 065010 (2013).
[6] H. Chen et al., Relativistic Quasimonoenergetic Positron Jets from Intense Laser-Solid Interactions, Phys. Rev. Lett. 105, 015003 (2010).

[7] H. Chen, S. C. Wilks, J. D. Bonlie, E. P. Liang, J. Myatt, D. F. Price, D. D. Meyerhofer, and P. Beiersdorfer, Relativistic Positron Creation Using Ultraintense Short Pulse Lasers, Phys. Rev. Lett. 102, 105001 (2009).

[8] H. Chen, S. C. Wilks, J. D. Bonlie, S. N. Chen, K. V. Cone, L. N. Elberson, G. Gregori, D. D. Meyerhofer, J. Myatt, D. F. Price, M. B. Schneider, R. Shepherd, D. C. Stafford, R. Tommasini, R. Maren, and P. Beiersdorfer, Making relativistic positrons using ultraintense short puls, Phys. Plasmas 16, 122702 (2009).

[9] G. Sarri, W. Schumaker, A. Di Piazza, M. Vargas, B. Dromey, M. E. Dieckmann, V. Chvykov, A. Maksimchuk, V. Yanovsky, Z. H. He, B. X. Hou, J. A. Nees, A. G. R. Thomas, C. H. Keitel, M. Zepf, and K. Krushelnick, A table-top laser-based source of short, collimated, ultrarelativistic positron beams, Proc. SPIE Int. Soc. Opt. Eng. 8779, 87790Z (2013).

[10] G. Sarri, W. Schumaker, A. Di Piazza, M. Vargas, B. Dromey, M. E. Dieckmann, V. Chvykov, A. Maksimchuk, V. Yanovsky, Z. H. He, B. X. Hou, J. A. Nees, A. G. R. Thomas, C. H. Keitel, M. Zepf, and K. Krushelnick, TableTop Laser-Based Source of Femtosecond, Collimated, Ultrarelativistic Positron Beams, Phys. Rev. Lett. 110, 255002 (2013).

[11] G. Sarri et al., Laser-driven generation of collimated ultrarelativistic positron beams, Plasma Phys. Controlled Fusion 55, 124017 (2013).

[12] G. Sarri et al., Generation of neutral and high-density electron-positron pair plasmas in the laboratory, Nat. Commun. 6, 6747 (2015).

[13] E. Liang, Gamma-ray and pair creation using ultra-intense lasers and astrophysical applications, High Energy Density Phys. 9, 425 (2013).

[14] R. Ruffini, G. Vereshchagin, and S. S. Xue, Electronpositron pairs in physics and astrophysics: From heavy nuclei to black holes, Phys. Rep. 487, 1 (2010).

[15] Y. Yan, Y. Wu, Z. Zhao, J. Teng, J. Yu, D. Liu, K. Dong, L. Wei, W. Fan, L. Cao, Z. Yao, and Y. Gu, Monte Carlo simulation study of positron generation in ultra-intense laser-solid interactions, Phys. Plasmas 19, 023114 (2012).

[16] J. Myatt, J. A. Delettrez, A. V. Maximov, D. D. Meyerhofer, R. W. Short, C. Stoeckl, and M. Storm, Optimizing electron-positron pair production on kilojoule-class high-intensity lasers for the purpose of pairplasma creation, Phys. Rev. E 79, 066409 (2009).

[17] S. C. Wilks, H. Chen, E. Liang, P. Patel, D. Price, B. Remington, R. Shepherd, M. Tabak, and W. L. Kruer, Electron-positron plasmas created by ultra-intense laser pulses interacting with solid targets, Astrophys. Space Sci. 298, 347 (2005).

[18] Y. H. Yan, Y. C. Wu, K. G. Dong, B. Zhang, Z. Q. Zhao, Z. Yao, and Y. Q. Gu, Simulation study of indirect positron generation by an ultra-short laser, Eur. Phys. J. D 68, 4 (2014).

[19] Y. H. Yan, B. Zhang, Y. C. Wu, K. G. Dong, Z. Yao, and Y. Q. Gu, Comparison of direct and indirect positrongeneration by an ultra-intense femtosecond laser, Phys. Plasmas 20, 103114 (2013). 
[20] Y. H. Yan, K. G. Dong, Y. C. Wu, B. Zhang, Z. Yao, and Y. Q. Gu, Numerical simulation study of positron production by intense laser-accelerated electrons, Phys. Plasmas 20, 103106 (2013).

[21] K. Nakashima and H. Takabe, Numerical study of pair creation by ultraintense lasers, Phys. Plasmas 9, 1505 (2002).

[22] J. Faure, C. Rechatin, A. Norlin, A. Lifschitz, Y. Glinec, and V. Malka, Controlled injection and acceleration of electrons in plasma wakefields by colliding laser pulses, Nature (London) 444, 737 (2006).

[23] N. A. M. Hafz, T. M. Jeong, I. W. Choi, S. K. Lee, K. H. Pae, V. V. Kulagin, J. H. Sung, T. J. Yu, K. H. Hong, T. Hosokai, J. R. Cary, D. K. Ko, and J. Lee, Stable generation of $\mathrm{GeV}$-class electron beams from self-guided laserplasma channels, Nat. Photonics 2, 571 (2008).

[24] S. Kneip et al., Near-GeV Acceleration of Electrons by a Nonlinear Plasma Wave Driven by a Self-Guided Laser Pulse, Phys. Rev. Lett. 103, 035002 (2009).

[25] S. Banerjee, S. Y. Kalmykov, N. D. Powers, G. Golovin, V. Ramanathan, N. J. Cunningham, K. J. Brown, S. Chen, I. Ghebregziabher, B. A. Shadwick, D. P. Umstadter, B. M. Cowan, D. L. Bruhwiler, A. Beck, and E. Lefebvre, Stable, tunable, quasimonoenergetic electron beams produced in a laser wakefield near the threshold for self-injection, Phys. Rev. ST Accel. Beams 16, 031302 (2013).

[26] W. P. Leemans, A. J. Gonsalves, H.-S. Mao, K. Nakamura, C. Benedetti, C. B. Schroeder, Cs. Tóth, J. Daniels, D. E. Mittelberger, S. S. Bulanov, J.-L. Vay, C. G. R. Geddes, and E. Esarey, Multi-GeV Electron Beams from Capillary-Discharge-Guided Subpetawatt Laser Pulses in the Self-Trapping Regime, Phys. Rev. Lett. 113, 245002 (2014).

[27] T. Tajima and J. M. Dawson, Laser Electron Accelerator, Phys. Rev. Lett. 43, 267 (1979).

[28] E. Esarey, C. B. Schroeder, and W. P. Leemans, Physics of laser-driven plasma-based electron accelerators, Rev. Mod. Phys. 81, 1229 (2009).

[29] A. Modena, Z. Najmudin, A. E. Dangor, C. E. Clayton, K. A. Marsh, C. Joshi, V. Malka, C. B. Darrow, C. Danson, D. Neely, and F. N. Walsh, Electron acceleration from the breaking of relativistic plasma waves, Nature (London) 377, 606 (1995).

[30] V. Malka, S. Fritzler, E. Lefebvre, M.-M. Aleonard, F. Burgy, J.-P. Chambaret, J.-F. Chemin, K. Krushelnick, G. Malka, S. P. D. Mangles, Z. Najmudin, M. Pittman, J.-P. Rousseau, J.-N. Scheurer, B. Walton, and A. E. Dangor, Electron acceleration by a wake field forced by an intense ultrashort laser pulse, Science 298, 1596 (2002).

[31] W. P. Leemans, P. Catravas, E. Esarey, C. G. R. Geddes, C. Toth, R. Trines, C. B. Schroeder, B. A. Shadwick, J. van Tilborg, and J. Faure, Electron-Yield Enhancement in a
Laser-Wakefield Accelerator Driven by Asymmetric Laser Pulses, Phys. Rev. Lett. 89, 174802 (2002).

[32] W. Lu, C. Huang, M. Zhou, M. Tzoufras, F. S. Tsung, W. B. Mori, and T. Katsouleas, A nonlinear theory for multidimensional relativistic plasma wave wakefields, Phys. Plasmas 13, 056709 (2006).

[33] W. Lu, C. Huang, M. Zhou, W. B. Mori, and T. Katsouleas, Nonlinear Theory for Relativistic Plasma Wakefields in the Blowout Regime, Phys. Rev. Lett. 96, 165002 (2006).

[34] W. Lu, M. Tzoufras, C. Joshi, F. S. Tsung, and W. B. Mori, Generating multi-GeV electron bunches using single stage laser wakefield acceleration in a 3D nonlinear regime, Phys. Rev. ST Accel. Beams 10, 061301 (2007).

[35] G.-Z. Sun, E. Ott, Y. C. Lee, and P. Guzdar, Self-focusing of short intense pulses in plasmas, Phys. Fluids 30, 526 (1987).

[36] L. Landau and G. Rumer, The cascade theory of electronic showers, Proc. R. Soc. A 166, 213 (1938).

[37] H. J. Bhabha and W. Heitler, The passage of fast electrons and the theory of cosmic showers, Proc. R. Soc. A 159, 432 (1937).

[38] B. Rossi and K. Greisen, Cosmic-ray theory, Rev. Mod. Phys. 13, 240 (1941).

[39] H. W. Koch and J. W. Motz, Bremsstrahlung cross-section formulas and related data, Rev. Mod. Phys. 31, 920 (1959).

[40] J. Joseph and F. Rohrlich, Pair production and Bremsstrahlung in the field of free and bound electrons, Rev. Mod. Phys. 30, 354 (1958).

[41] C. M. Davisson and R. D. Evans, Gamma-ray absorption coefficients, Rev. Mod. Phys. 24, 79 (1952).

[42] B. Rossi, High-Energy Particles (Prentice-Hall, Englewood Cliffs, New Jersey, 1952).

[43] Heitler, The Quantum Theory of Radiation (Oxford University, New York, 1936).

[44] O. Klein and Y.Z. Nishina, About the scattering of radiation by free electrons after the new relativistic quantum dynamics of Dirac, Z. Phys. 52, 853 (1929).

[45] P. Marmier and E. Sheldon, Physics of Nuclei and Particles (Academic, New York, 1969).

[46] T. J. Yu, S. K. Lee, J. H. Sung, J. W. Yoon, T. M. Jeong, and J. Lee, Generation of high-contrast, 30 fs, $1.5 \mathrm{PW}$ laser pulses from chirped-pulse amplification Ti:sapphire laser, Opt. Express 20, 10807 (2012).

[47] E. W. Gaul, M. Martinez, J. Blakeney, A. Jochmann, M. Ringuette, D. Hammond, T. Borger, R. Escamilla, S. Douglas, W. Henderson, G. Dyer, A. Erlandson, R. Cross, J. Caird, C. Ebbers, and T. Ditmire, Demonstration of a 1.1 petawatt laser based on a hybrid optical parametric chirped pulse amplification/mixed Nd:glass amplifier, Appl. Opt. 49, 1676 (2010).

[48] R. Dabu, High power femtosecond lasers at ELI-NP, AIP Conf. Proc. 1645, 219 (2015). 